\title{
Proton Pump Inhibitor Use Increases Pyogenic Liver Abscess Risk: A Nationwide Cohort Study
}

\author{
Joo Hyun Oh, ${ }^{1,2}$ Danbee Kang, ${ }^{3,4}$ Wonseok Kang, ${ }^{1}$ Eliseo Guallar, ${ }^{4,5}$ Juhee Cho, ${ }^{3,4,5}$ and Yang Won Min ${ }^{1 *}$ \\ ${ }^{I}$ Department of Medicine, Samsung Medical Center, Sungkyunkwan University School of Medicine, Seoul, Korea; ${ }^{2}$ Department of Medicine, \\ Nowon Eulji Medical Center, Eulji University School of Medicine, Seoul, Korea; ${ }^{3}$ Department of Clinical Research Design and Evaluation, \\ Samsung Advanced Institute for Health Science and Technology, Sungkyunkwan University School of Medicine, Seoul, Korea; ${ }^{4}$ Center for \\ Clinical Epidemiology, Samsung Medical Center, Sungkyunkwan University, Seoul, Korea; and ${ }^{5}$ Departments of Epidemiology and Medicine and \\ Welch Center for Prevention, Epidemiology and Clinical Research, Johns Hopkins Medical Institutions, Baltimore, Maryland, USA
}

\section{Background/Aims}

Proton pump inhibitors (PPIs) increase gastric pH and alter the gut microbiome. An increased risk for infectious diseases has been reported in PPI users. However, little is known about the association of PPI use with pyogenic liver abscess (PLA) incidence risk.

\section{Methods}

We conducted a population-based cohort study using data from a nationwide representative sample of the Korean general population followed up for 10 years (January 1, 2003 to December 31, 2013). We identified PPI prescriptions and considered PPI as a timevarying variable. Proportional hazards regression model was used for incident PLA comparing PPI use versus non-use. Propensity score matching was also conducted.

\section{Results}

During the 4209229 person-years of follow-up, 58595 participants had at least 1 PPI prescription and 541 patients developed liver abscess. The age-, sex-, residential area-, and income-adjusted hazard ratio for PLA incidence with PPI use was 4.19 (95\% Cl, 2.546.92). The association was observed in fully adjusted models (hazard ratio 3.88; $95 \% \mathrm{Cl}, 2.33-6.44$ ). The positive association between PPI use and PLA was consistent in all subgroups analyzed and in propensity score matching group.

\section{Conclusion}

The present data indicate that PPI use is associated with an increased PLA risk. Therefore, it is necessary to prescribe PPIs with clear indication and to avoid improper use of PPIs.

\section{(J Neurogastroenterol Motil 2021;27:555-564)}

\section{Key Words}

Cohort studies; Gastrointestinal microbiome; Liver abscess, pyogenic; Proton pump inhibitors

Received: September 27, 2020 Revised: February 18, 2021 Accepted: March 21, 2021

(.) This is an Open Access article distributed under the terms of the Creative Commons Attribution Non-Commercial License (http://creativecommons. org/licenses/by-nc/4.0) which permits unrestricted non-commercial use, distribution, and reproduction in any medium, provided the original work is properly cited.

*Correspondence: Yang Won Min, MD, PhD

Department of Medicine, Samsung Medical Center, Sungkyunkwan University School of Medicine, 81 Irwon-ro, Gangnam-gu, Seoul 06351, Korea

Tel: +82-2-3410-3409, Fax: +82-2-3410-6983, E-mail: yangwonee@gmail.com

Joo Hyun Oh and Danbee Kang contributed equally to this study. 


\section{Introduction}

Proton pump inhibitors (PPIs) effectively block gastric acid secretion by irreversibly binding to and inhibiting the hydrogenpotassium ATPase pump that resides on the luminal surface of the parietal cell membrane. ${ }^{1}$ They currently play a crucial role in the management of peptic ulcer disease, gastroesophageal reflux disease (GERD), Zollinger-Ellison syndrome, nonsteroidal anti-inflammatory drug-associated ulcers, and eradication of Helicobacter pylori. PPIs are generally viewed as safe drugs, but their long-term use has been associated with several safety concerns, particularly infectious diseases. ${ }^{2}$ For instance, PPI association with an increased Clostridium difficile infection risk ${ }^{3,4}$ and spontaneous bacterial peritonitis in patients with liver cirrhosis ${ }^{5}$ have been reported.

Pyogenic liver abscess (PLA) is a relatively uncommon illness that has been associated with significant morbidity, mortality, and healthcare resource consumption. ${ }^{6}$ It may arise from hematogenous spread of bacteria or local spread from adjacent sites of infection within the peritoneal cavity. Although appendicitis with rupture was the most common source in the past, biliary tract-associated disease is currently the most common source.

Previously, a large cohort study reported that PPIs are associated with cholangitis and explained that PPI can increase the risk of inducing an imbalance of specific bacterial pathogens in the biliary microbiota. ${ }^{8}$ Since cholangitis is a major risk factor of PLA, PPI use is expected to be associated with PLA. Previous case-control studies from Taiwan have reported an association between PPI use and PLA development. ${ }^{9,10}$ However, these studies are susceptible to selection bias and impossible to comment on the temporal relationship between PPI use and PLA. To the best of our knowledge, no longitudinal study with carefully adjusted variables has investigated the impact of PPI on PLA till date. Thus, we evaluated whether PPI use increases PLA risk using a large population cohort.

\section{Materials and Methods}

\section{Study Population and Design}

The National Health Insurance Service-National Sample Cohort (NHIS-NSC) is a population-based retrospective cohort consisting of a representative sample of $2.2 \%$ Korean citizens enrolled in the NHIS. ${ }^{11}$ The NHIS is the universal single-payer national healthcare system of Korea. NHIS covers all regions of Korea and maintains national records of all insurance-covered in- and outpatient visits, procedures, and prescriptions. NHIS-NSC sampling consisted of a systematic stratified random sampling with proportional allocation within each stratum. The sampling procedures and representativeness of the cohort have been described in detail in a previous study. ${ }^{11}$ In Korea, the NHIS also provides free annual or biennial health screening exams assessing cardiovascular and diabetes risk factors, including smoking and alcohol drinking habits, to all insured subjects. Approximately $72 \%$ eligible beneficiaries undergo such screening exams. ${ }^{12}$

We used the person-level longitudinal NHIS-NSC registration, claim, and health screening exam data recorded between January 1, 2003 and December 31, 2013. ${ }^{11}$ Our study population included all men and women $\geq 20$ years old participating in the NHIS-NSC cohort with at least 1 health screening between January 1, 2003 and December 31, 2013 ( $\mathrm{n}=588$ 326). We then excluded the participants with PLA $(n=201)$ or history of cholangiocarcinoma, gallbladder (GB) cancer, pancreas cancer, or liver cancer $(n=2241)$ between January 1, 2003 and the baseline screening exam. In addition, we excluded participants who had consumed PPIs within 180 days before the baseline screening exam $(\mathrm{n}=2426)$. The final sample consisted of 583538 participants (290 814 men and 292724 women; Fig. 1). We also generated propensity score (PS) matching cohort based on age and comorbidities. The PS matching resulted in 47362 patients who received PPIs $(n=23681)$ or unexposed group $(n=23681)$. The institutional

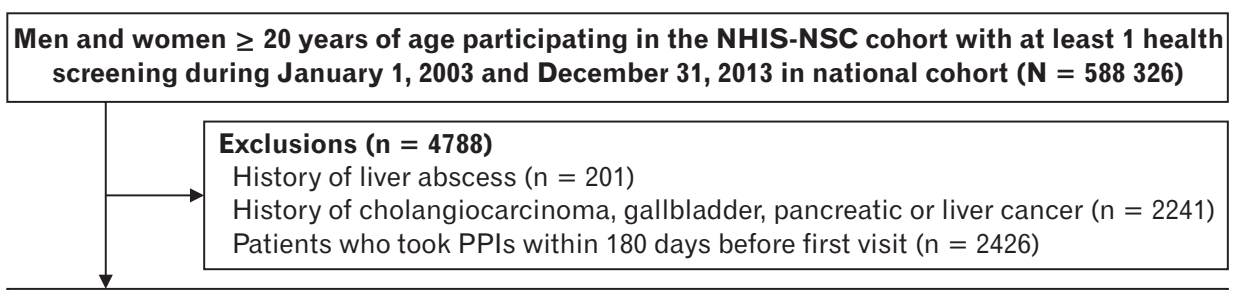

Participants included in this study $(n=583538)$
Figure 1. Flowchart of study participants. NHIS-NSC, National Health Insurance Service-National Sample Cohort. PPIs, proton pump inhibitors. 
review board approved this study and waived the requirement for informed consent, as we used only de-identified data (IRB No. 2020-03-118).

\section{Definition of Proton Pump Inhibitor Exposure}

HIS claims for in- and out-patient visits, procedures, and prescriptions were coded using the International Classification of Diseases, 10th Revision (ICD-10), adopted in Korea in 1995, and the Korean Drug and Anatomical Therapeutic Chemical Codes. ${ }^{13,14}$

PPI is available only by prescription and cannot be purchased over the counter in Korea. PPI use was identified as prescriptions with Korean Drug and Anatomical Therapeutic Chemical Codes $\mathrm{A} 02 \mathrm{BC} 01, \mathrm{~A} 02 \mathrm{BC} 02, \mathrm{~A} 02 \mathrm{BC} 03, \mathrm{~A} 02 \mathrm{BC} 04$, and $\mathrm{A} 02 \mathrm{BC} 05$ and considered as a time-varying variable to control immortal time bias. ${ }^{15,16}$ To avoid reverse causation bias occurring if PPI prescription was a consequence of symptoms caused by PLA, we considered that PPI use could not be responsible for PLA cases occurring in the first seven days after initiating PPI treatment based on a previous study. ${ }^{17}$

Moreover, as the effects of PPI on the microbiome may persist even after medication use, for each PPI prescription, we considered that the patient continued to be potentially affected by PPIs for 60 days after the prescription expiry date (that is, we assumed that the effect of PPIs had a residual effect that persisted for 60 days after using the medication). If a new prescription was redeemed $<60$ days after the expiry of a prior prescription, the gap was considered also an exposure period (continuing PPI treatment). If a new prescription was redeemed $\geq 60$ days after the expiry of a previous prescription, the gap was coded as a non-exposure period between 2 different PPI treatments. In sensitivity analysis, we repeated the analyses assuming that the period of PPI residual effect after using the medication was 30 days or 90 days, instead of 60 days.

\section{Outcome Definition}

The study outcome was PLA development, defined as a new ICD-10 code K75.0 in any hospitalization or out-patient visit claims during follow-up. As the NHIS routinely audits the claims, such data are considered reliable and have been used in numerous peerreviewed studies. ${ }^{11,18-20}$ A previous validation study of discharge diagnoses in the NHIS database compared with medical records found an overall positive predictive value of $80.24 \% .{ }^{11}$

\section{Other Variables}

For each participant, the time of first health screening exam during the study period was considered the study baseline. Infor- mation on age, sex, residential area, and income level was obtained from the NHIS insurance eligibility database for the year of baseline health screening exam. The number of out-patient clinic visits for each calendar year was obtained from medical treatments database. Information on smoking and drinking habits and body mass index (BMI) was obtained from the baseline health screening exam. The health screening exam database included questionnaire information on smoking status, alcohol intake frequency, and height, weight, and blood pressure measurements. ${ }^{11} \mathrm{BMI}$ was calculated as weight $(\mathrm{kg})$ divided by height $(\mathrm{m})$ squared.

Information on hepatobiliary comorbidities and the use of immune-suppressants, antibiotics, and systemic corticosteroids throughout follow-up was obtained from the medical treatments database. We considered the following hepatobiliary and upper gastrointestinal diseases that could influence PLA risk: GB stones (K80.2), common bile duct/intrahepatic bile duct stones (K80.5), biliary stricture/atresia (K83.1, Q44.3, Q44.2), biliary cysts (K83.5, Q44.4), liver cirrhosis (K70.30, K70.31, K74.60-K74.62, K74.69, K74.1, K74.10-K74.12, K74.19), GERD (K21), gastric ulcer (K25), and duodenal ulcer (K26). Use of immune-suppressants, antibiotics, and systemic corticosteroids throughout follow-up was identified by Korean Drug and Anatomical Therapeutic Chemical Codes (Supplementary Table 1). Other comorbidities were adjusted using Charlson comorbidity index $(\mathrm{CCI}) .{ }^{21} \mathrm{CCI}$ is the most widely used comorbidity index. It contains 10 issues including diabetes, congestive heart failure, peripheral vascular disease, chronic pulmonary disease, mild and severe liver disease, hemiplegia, renal disease, leukemia, lymphoma, metastatic tumor, and acquired immunodeficiency syndrome. ${ }^{22}$

\section{Statistical Methods}

The study endpoint was PLA development. Participants contributed follow-up person-time from the date of baseline health screening exam until PLA development, death, or the end of study period (December 31, 2013), whichever was first. The study exposure was PPI use, considered as a time-varying variable. For each PPI treatment course, participants who took PPIs contributed person-time to the exposed group starting seven days after the date of initial prescription until 60 days after date of expiry of last prescription. Unexposed person-time was contributed by participants who did not take PPIs and by participants who had PPIs while they were not taking them. Data regarding PPI prescriptions dispended after the patient had developed liver abscess were not included in the study, as study follow-up ended with the first episode of liver abscess during the study period. Detailed methods of the study have 
Table 1. Characteristics of Study Participants ( $\mathrm{N}=583$ 538)

\begin{tabular}{|c|c|c|c|}
\hline \multirow{2}{*}{ Characteristic } & \multicolumn{2}{|c|}{ At least 1 PPI prescription during follow-up } & \multirow{2}{*}{$P$-value } \\
\hline & No $(\mathrm{n}=524943)$ & Yes $(n=58595)$ & \\
\hline Sex & & & 0.001 \\
\hline Male & $261999(49.9)$ & $28815(49.2)$ & \\
\hline Female & $262944(50.1)$ & $29780(50.8)$ & \\
\hline Age (yr) & & & $<0.001$ \\
\hline $20-29$ & $107361(20.5)$ & $4524(7.7)$ & \\
\hline $30-39$ & $92564(17.6)$ & $6467(11.0)$ & \\
\hline $40-49$ & $148760(28.3)$ & $15827(27.0)$ & \\
\hline $50-59$ & $89882(17.1)$ & $14069(24.0)$ & \\
\hline $60-69$ & $56347(10.7)$ & $11426(19.5)$ & \\
\hline $70-79$ & $24604(4.7)$ & $5431(9.3)$ & \\
\hline$\geq 80$ & $5425(1.0)$ & $851(1.5)$ & \\
\hline Income percentile & & & $<0.001$ \\
\hline$\leq 30$ th & $140892(26.8)$ & $14731(25.1)$ & \\
\hline$>30$ th $-\leq 70$ th & $162450(31.0)$ & $15879(27.1)$ & \\
\hline$>70$ th & $221601(42.2)$ & $27985(47.8)$ & \\
\hline Residential area & & & $<0.001$ \\
\hline Metropolitan & $352847(67.2)$ & $36305(62.0)$ & \\
\hline Rural & $172096(32.8)$ & $22290(38.0)$ & \\
\hline $\mathrm{BMI}\left(\mathrm{kg} / \mathrm{m}^{2}\right)$ & & & $<0.001$ \\
\hline Underweight $(<18.5)$ & $25316(4.8)$ & $2320(4.0)$ & \\
\hline Normal $(\geq 18.5-<23)$ & $220094(41.9)$ & $22015(37.6)$ & \\
\hline Overweight $(\geq 23-<25)$ & $121540(23.2)$ & $14587(24.9)$ & \\
\hline Obese $(\geq 25)$ & $157703(30.0)$ & $19638(33.5)$ & \\
\hline Unknown & $290(0.1)$ & $35(0.1)$ & \\
\hline Smoking status & & & $<0.001$ \\
\hline Never & $329376(62.8)$ & $37509(64.0)$ & \\
\hline Past & $30359(5.8)$ & $3046(5.2)$ & \\
\hline Current & $134378(25.6)$ & $13727(23.4)$ & \\
\hline Unknown & $30830(5.9)$ & $4313(7.4)$ & \\
\hline Alcohol intake (times per week) & & & $<0.001$ \\
\hline$<1$ & $330849(63.0)$ & $40078(68.4)$ & \\
\hline $1-2$ & $127270(24.2)$ & $10355(17.7)$ & \\
\hline $3-4$ & $38863(7.4)$ & $4148(7.1)$ & \\
\hline Almost every day & $17093(3.3)$ & $2649(4.5)$ & \\
\hline Unknown & $10868(2.1)$ & $1365(2.3)$ & \\
\hline Number of clinic visits ${ }^{a}$ & $4(1-10)$ & $8(3-16)$ & $<0.001$ \\
\hline Charlson comorbidity index & & & $<0.001$ \\
\hline 0 & $422563(80.5)$ & $40812(69.7)$ & \\
\hline 1 & $80168(15.3)$ & $12700(21.7)$ & \\
\hline$\geq 2$ & $22212(4.2)$ & $5083(8.7)$ & \\
\hline Hepatobiliary diseases ${ }^{\mathrm{b}}$ & $7795(1.5)$ & $1538(2.6)$ & $<0.001$ \\
\hline GB stone & $3566(0.7)$ & $545(0.9)$ & $<0.001$ \\
\hline $\mathrm{CBD} / \mathrm{IHD}$ stone & $908(0.2)$ & $160(0.3)$ & $<0.001$ \\
\hline Biliary stricture/atresia & $382(0.1)$ & $61(0.1)$ & $<0.001$ \\
\hline
\end{tabular}


Table 1. Continued

\begin{tabular}{|c|c|c|c|}
\hline \multirow{2}{*}{ Characteristic } & \multicolumn{2}{|c|}{ At least 1 PPI prescription during follow-up } & \multirow{2}{*}{$P$-value } \\
\hline & No $(\mathrm{n}=524943)$ & Yes $(n=58595)$ & \\
\hline Hepatobiliary diseases $^{\mathrm{b}}$ & & $1538(2.6)$ & $<0.001$ \\
\hline Biliary cyst & $56(0.0)$ & $7(0.0)$ & 0.778 \\
\hline Liver cirrhosis & $3362(0.6)$ & $862(1.5)$ & $<0.001$ \\
\hline Upper gastrointestinal diseases $^{\mathrm{b}}$ & $182297(34.7)$ & $33634(57.4)$ & $<0.001$ \\
\hline Gastroesophageal reflux disease & $110095(21.0)$ & $20108(34.2)$ & $<0.001$ \\
\hline Gastric ulcer & $68146(13.0)$ & $12909(22.0)$ & $<0.001$ \\
\hline Duodenal ulcer & $14995(2.9)$ & $3195(5.5)$ & $<0.001$ \\
\hline
\end{tabular}

${ }^{a}$ During the previous year.

${ }^{b}$ Reported at least once during follow-up.

PPI, proton pump inhibitor; BMI, body mass index; GB, gall bladder; CBD, common bile duct; IHD, intrahepatic bile duct.

Values are presented as $\mathrm{n}(\%)$ or median (range).

been described in our previous study. ${ }^{8}$

We used a proportional hazards regression model to estimate the hazard ratios (HRs) with 95\% CIs for liver abscess incidence with PPI use versus that with non-use. We used 3 models with increasing degrees of adjustment to account for potential confounding factors. Model 1 was adjusted for age (5-year categories), sex, residential area (metropolitan and rural), income percentile ( $\leq 30$ th, $>$ 30th $-\leq 70$ th, $>70$ th percentiles $)$, BMI $(<18.5, \geq 18.5-<23$, $\geq 23-<25, \geq 25 \mathrm{~kg} / \mathrm{m}^{2}$, and unknown), smoking status (never, former, current, and unknown), alcohol intake frequency $(<1,1-2$, 3-4 times per week, almost every day, and unknown), and CCI $(0,1$, and $\geq 2)$ at the baseline health screening exam as time-fixed variables. Model 2 was further adjusted for the number of out-patient clinic visits during the year prior to baseline health screening exam as a time-fixed variable, development of hepatobiliary diseases (GB stones, common bile duct/intrahepatic bile duct stones, biliary stricture/atresia, biliary cysts, liver cirrhosis), upper gastrointestinal diseases (GERD, gastric ulcer, duodenal ulcer), and use of immunesuppressants, antibiotics, and systemic corticosteroids over followup as time-varying variables. The periods were measured from the index date to the date of development of PLA or the last follow-up. We examined the proportional hazards assumption using plots of the $\log (-\log )$ survival function and Schoenfeld residuals.

In addition, we performed stratified analyses to evaluate the association of PPI use with liver abscess incidence in pre-specified subgroups defined by age ( $<65$ years and $\geq 65$ years), sex, income percentiles ( $\leq 30 \mathrm{th},>30$ th- $\leq 70 \mathrm{th},>70$ th percentiles), residential area (metropolitan and rural), obesity (no and yes), smoking status (never and ever), alcohol intake ( $<1$ time per week, and $\geq 1$ time per week), CCI ( 0 and $\geq 1)$, and number of out-patient clinic visits during the year prior to baseline health screening exam $(<5$ and $\geq 5$ ).

All analyses were performed using STATA version 15 (StataCorp LP; College Station, TX, USA).

\section{Results}

The 58595 (10.0\%) participants had at least 1 PPI prescription during the 4209229 person-years of follow-up. Compared to nonusers, PPI users were older and more likely to have comorbidities at the start of the follow-up. PPI users were also more likely to have hepatobiliary diseases (1.5\% vs $2.6 \%)$ and upper gastrointestinal diseases (34.7\% vs 57.3\%) over follow-up than non-users (Table 1). The 1:1 PS-matched analysis generated 23681 pairs, and the baseline characteristics of the 2 groups were described in Table 2 . The 2 groups were comparable for age, $\mathrm{BMI}$, and CCI.

The number of PLA incidences observed over the follow-up during unexposed and PPI-exposure periods were 525 (incidence rate: 13 cases per 100000 person-years) and 16 (incidence rate: 88 cases per 100000 person-years), respectively (Table 3 and Fig. 2).

The age-, sex-, residential area-, income percentile-, BMI-, smoking-, and alcohol intake-adjusted HR for PLA incidence with PPI use vs that with no PPI use was 4.19 (95\% CI, 2.54-6.92). After adjusting for multiple confounders, the association remained intact (fully-adjusted HR, 3.88; 95\% CI, 2.33-6.44). Furthermore, with the sensitivity analysisbased on the duration of period of residual effect, the results using 30 days (fully-adjusted HR, 3.75; 95\% CI, 2.48-5.67) and 90 days (fully-adjusted HR, 3.69; 95\% CI, 2.31-5.90) of residual-effect period were similar to those using 60 days (Supplementary Table 2). Finally, when we evaluated whether the association between PPI and liver abscess differed in pre-specified subgroups, the positive association between PPI and 
Table 2. Characteristics of Study Participants in Propensity Matched Group ( $\mathrm{N}=47$ 362)

\begin{tabular}{|c|c|c|c|}
\hline \multirow{2}{*}{ Characteristic } & \multicolumn{2}{|c|}{ At least 1 PPI prescription during follow-up } & \multirow{2}{*}{$P$-value } \\
\hline & No $(n=23681)$ & Yes $(n=23681)$ & \\
\hline Sex & & & 0.416 \\
\hline Male & $10647(45.0)$ & $10558(44.6)$ & \\
\hline Female & $13035(55.0)$ & $13123(55.4)$ & \\
\hline Age (yr) & & & $>0.99$ \\
\hline $20-29$ & $862(3.6)$ & $862(3.6)$ & \\
\hline $30-39$ & $1577(6.7)$ & $1577(6.7)$ & \\
\hline $40-49$ & $5367(22.7)$ & $5367(22.7)$ & \\
\hline $50-59$ & $6086(25.7)$ & $6086(25.7)$ & \\
\hline $60-69$ & $6122(25.9)$ & $6122(25.9)$ & \\
\hline $70-79$ & $3714(13.4)$ & $3174(13.4)$ & \\
\hline$\geq 80$ & $493(2.1)$ & $493(2.1)$ & \\
\hline $\operatorname{BMI}\left(\mathrm{kg} / \mathrm{m}^{2}\right)$ & & & 0.916 \\
\hline Underweight $(<18.5)$ & $863(3.6)$ & $897(3.8)$ & \\
\hline Normal $(\geq 18.5-<23)$ & $8438(35.6)$ & $8416(35.5)$ & \\
\hline Overweight $(\geq 23-<25)$ & $5910(25.0)$ & $5897(24.9)$ & \\
\hline Obese $(\geq 25)$ & $8451(35.7)$ & $8455(35.7)$ & \\
\hline Unknown & $19(0.1)$ & $16(0.1)$ & \\
\hline Charlson comorbidity index & & & $>0.99$ \\
\hline 0 & $5898(24.9)$ & $5898(24.9)$ & \\
\hline 1 & $12700(53.6)$ & $12700(53.6)$ & \\
\hline$\geq 2$ & $5083(21.5)$ & $5083(21.5)$ & \\
\hline \multicolumn{4}{|l|}{ Hepatobiliary diseases $^{a}$} \\
\hline GB stone & $237(1.0)$ & $257(1.1)$ & 0.366 \\
\hline $\mathrm{CBD} / \mathrm{IHD}$ stone & $49(0.2)$ & $85(0.4)$ & 0.002 \\
\hline Biliary stricture/atresia & $45(0.2)$ & $32(0.1)$ & 0.138 \\
\hline Biliary cyst & $4(0.0)$ & $4(0.0)$ & $>0.99$ \\
\hline Liver cirrhosis & $266(1.1)$ & $423(1.8)$ & $<0.001$ \\
\hline
\end{tabular}

${ }^{a}$ Reported at least once during follow-up.

PPI, proton pump inhibitor; BMI, body mass index; GB, gallbladder; CBD, common bile duct; IHD, intrahepatic bile duct.

Values are presented as $\mathrm{n}(\%)$.

Table 3. Hazard Ratios (95\% Confidence Intervals) for Pyogenic Liver Abscess Incidence Associated With of Proton Pump Inhibitor Use (N = $583538)$

\begin{tabular}{|c|c|c|c|c|c|}
\hline & \multirow{2}{*}{ Person-years } & \multirow{2}{*}{ No. of cases } & \multirow{2}{*}{$\begin{array}{c}\text { Incidence rate } \\
\text { (per } 100000 \text { py) }\end{array}$} & \multicolumn{2}{|c|}{ Hazard ratio $(95 \% \mathrm{CI})$} \\
\hline & & & & Model 1 & Model 2 \\
\hline \multicolumn{6}{|c|}{ PPI prescription plus 60 days of residual-effect period } \\
\hline Unexposed period & 4191085 & 525 & 13 & Reference & Reference \\
\hline Use of PPI period & 18144 & 16 & 88 & $4.19(2.54-6.92)$ & $3.88(2.33-6.44)$ \\
\hline$P$-value & & & & $<0.01$ & $<0.01$ \\
\hline
\end{tabular}

py, person year; PPI, proton pump inhibitor.

Model 1: Adjusted for age, sex, residential area, and income level, body mass index, smoking status, alcohol intake frequency, and Charlson comorbidity index at the start of follow-up as time-fixed variables.

Model 2: Further adjusted for the number of clinic visits during the year prior to the beginning of follow-up as time-fixed variable, development of hepatobiliary diseases, and upper gastrointestinal disease, and use of immune-suppressants, antibiotics, and systemic corticosteroids over follow-up as time-varying variables. 


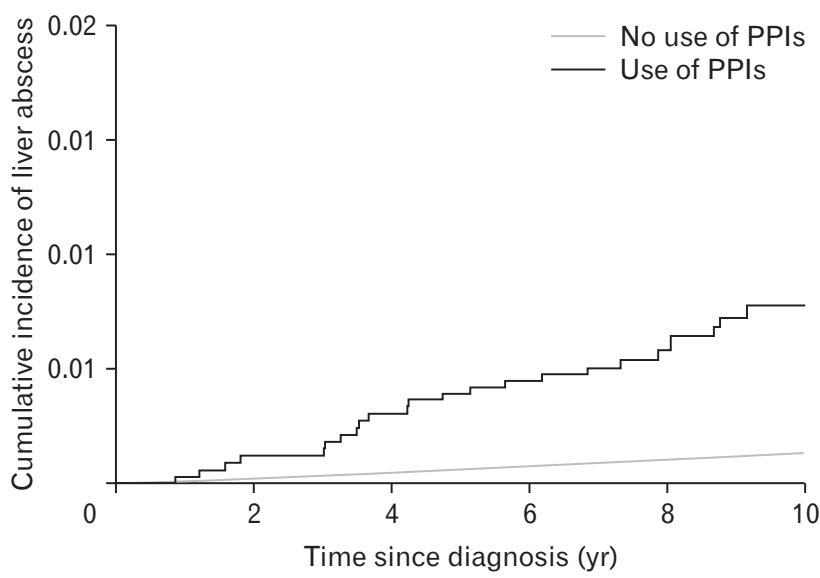

Figure 2. Cumulative proportional incidence of pyogenic liver abscess by proton pump inhibitors (PPIs). liver abscess was consistent in all subgroups analyzed (all $P$-values for interaction $>0.10$; Fig. 3).

In PS matching cohort, the results after PS matching were similar to the results before matching. The incidences of PLA development were 65 in unexposed period (incidence rate: 20 cases per 100000 person-years) and 11 in exposed period (incidence rate: 135 cases per 100000 person-years). Multivariate analysis also showed that PPI use was independently associated with PLA development (Table 4).

The cumulative median duration of PPI in each PPI prescription period was 10 days (interquartile range $[\mathrm{IQR}]=7,16$ ). Among the participants who developed PLA, the cumulative median duration of PPI was 10 days $(\mathrm{IQR}=4,25.5)$. There were no significant differences between median duration of PPI by patients
Subgroup

Age (yr)
$<65(\mathrm{n}=521085)$
$\geq 65(\mathrm{n}=62453)$
$P$-value
Sex
Male $(\mathrm{n}=290814)$
Female $(\mathrm{n}=292724)$
$P$-value
Income percentile
$\leq 30$ th $(\mathrm{n}=155623)$
$>30$ th- $\leq 70$ th $(\mathrm{n}=178329)$
$>70$ th $(\mathrm{n}=249586)$
$P$-value
Residential area
Metropolitan $(\mathrm{n}=389152)$
Rural $(\mathrm{n}=194386)$
$P$-value
Obese
No $(\mathrm{n}=405872)$
Yes $(\mathrm{n}=177341)$
$P$-value
Smoking status
Never $(\mathrm{n}=366885)$
Ever $(\mathrm{n}=181510)$
$P$-value
Frequency of alcohol intake per
$<1$ time $(\mathrm{n}=370927)$
More than 1 time $(\mathrm{n}=200378)$
$P$-value
Chalue
0 -value
Orall $(\mathrm{n}=463375)$

HR for incident liver abscess (95\% CI)

$4.83(2.69,8.67)$

$2.42(0.89,6.56)$

0.240

$4.26(2.32,7.83)$

$3.23(1.32,7.91)$

0.620

$2.07(0.51,8.40)$

$5.68(2.49,12.95)$

$3.80(1.87,7.73)$

0.460

$4.73(2.50,8.96)$

$2.97(1.31,6.72)$

0.370

$2.83(1.33,6.03)$

$5.43(2.76,10.66)$

0.200

$3.06(1.51,6.23)$

$5.97(2.78,12.82)$

0.210

$3.04(1.56,5.95)$

$6.45(3.00,13.85)$

0.140

$3.36(1.58,7.14)$

$4.64(2.36,9.15)$

0.530

$6.72(2.75,16.43)$

$3.19(1.74,5.87)$

0.170

$3.88(2.33,6.44)$

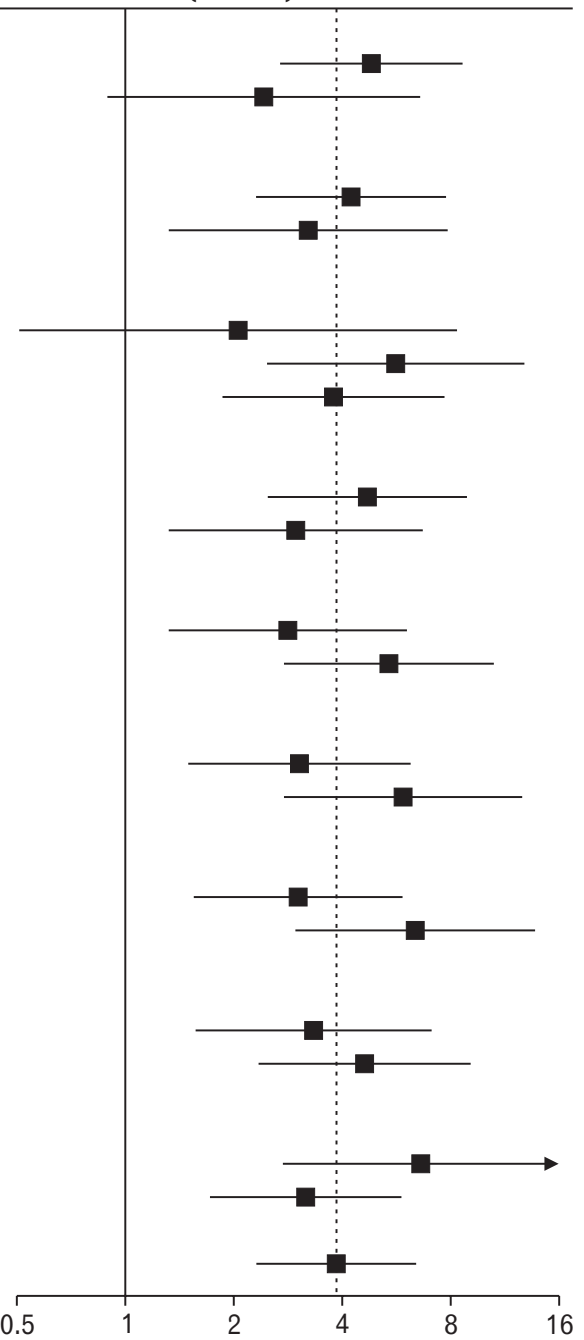

Figure 3. Hazard ratios (HRs) (95\% CI) for incident pyogenic liver abscess associated with the use of proton pump inhibitor in selected population sub- 
Table 4. Hazard Ratios (95\% Confidence Intervals) for Incident Liver Abscess Associated With Use of Proton Pump Inhibitors in Propensity Matched Group $(\mathrm{N}=47362)$

\begin{tabular}{|c|c|c|c|c|c|}
\hline & \multirow{2}{*}{ Person-years } & \multirow{2}{*}{ No. of cases } & \multirow{2}{*}{$\begin{array}{l}\text { Incidence rate } \\
\text { (per } 100000 \text { py) }\end{array}$} & \multicolumn{2}{|c|}{ Hazard ratio $(95 \% \mathrm{CI})$} \\
\hline & & & & Model 1 & Model 2 \\
\hline \multicolumn{6}{|l|}{ PPI prescription } \\
\hline Unexposed period & 321283 & 65 & 20 & Reference & Reference \\
\hline Use of PPI period & 8128 & 11 & 135 & $6.02(3.16-11.49)$ & $4.81(2.48-9.35)$ \\
\hline$P$-value & & & & $<0.01$ & $<0.01$ \\
\hline
\end{tabular}

py, person year; PPI, proton pump inhibitor.

The participants were matched with age and comorbidities.

Model 1: Adjusted for sex, residential area, and income level, body mass index, smoking status, and frequency of alcohol intake at the start of follow-up as time-fixed variables.

Model 2: Further adjusted for the number of clinic visits during the year prior to the beginning of follow-up as time-fixed variable, development of hepatobiliary diseases, of upper gastrointestinal disease, and the use of immune-suppressants, antibiotics, and systemic corticosteroids over follow-up as time-varying variables.

with and without development of PLA (patient without PLA [me$\operatorname{dian}\{$ IQR $\}: 10[7,16]$ vs patients with PLA $[$ median $\{$ IQR $\}$ : $10(4,25.5): P=0.983)$. The HR for PLA related with PPI use was 2.59 (95\% CI, 1.15-5.84) when we conducted a sensitivity analysis that PPI exposure was defined as patients who used PPI for more than 14 days (Supplementary Table 3).

\section{Discussion}

In this nationwide population-based study, PPI prescriptions were significantly associated with an increased PLA rate across all subgroups as well as after adjustment for confounding factors. Since the unexposed group was demographically favorable, we used PS matching to balance baseline features. The positive correlation between PPI usage and PLA development maintained after matching. To the best of our knowledge, the present study is the first cohort study to demonstrate a significant association between PPI use and PLA risk.

PPIs are commonly prescribed drugs in every day clinical practice and generally thought to be drugs with a great safety profile. However, widespread PPI use has led to concerns about the adverse events, particularly infection risks. Recently, 2 populationbased case-control studies showed an association between PPI use and increased PLA risk. ${ }^{9,10}$ However, direct evidence regarding the contribution of PPI use to PLA development is limited because these studies were case-control studies and some potential factors, such as comorbidities, drug history, and biliary stricture and cysts, were not fully adjusted. In this present study, we carefully adjusted hepatobiliary diseases, use of immune-suppressants, antibiotics, and systemic corticosteroids, and comorbidities. In addition, we excluded the first 7 days of PPI prescription from the definition of exposure periods to reduce reverse causation bias because a previous study reported that the mean duration of symptoms before admission was 5.5 days. In multivariate and subgroup analyses, we found a strong association between PPI use and PLA development.

PLA source includes ascending biliary tract infection, portal bacteremia, septicemia, direct extension from intraperitoneal infection, direct trauma to the liver, and secondary infection of metastatic cancer. $^{23}$ PPI exposure can be one of the main reasons leading to PLA because PPIs induce not only the alteration of gut microbiome, but also morphological changes in the bile duct. Gastric acidity is a major defense mechanism of the body, which sterilizes contents entering the digestive tract, prevents bacterial colonization in the gastrointestinal tract, and influences the normal intestinal flora composition. ${ }^{24}$ Contrarily, PPIs directly block gastric acid secretion by irreversibly binding to and inhibiting the hydrogen-potassium ATPase pump that resides on the luminal surface of the parietal cell membrane. ${ }^{25}$ Systematic review and meta-analysis demonstrated that PPIs may disrupt the gut ecology and alter the bacterial growth, ranging from abnormal bacterial counts to overt small intestine bacterial overgrowth. ${ }^{26,27}$ Several studies have demonstrated that even short-term PPI use (less than 2 weeks) can alter the gut microbiome. ${ }^{28-30}$ The colonization is anticipated to precede intestinal mucosal invasion and portal venous flow or ascending biliary infection. ${ }^{31}$ In addition, an animal model study has shown that 30-day PPI-exposed rats experienced changes in bile duct including ductal epithelial proliferation, micropapillary growth of biliary epithelium, focal bile duct stricture formation, and bile duct obstruction. ${ }^{32}$ These conditions potentially increase the susceptibility to biliary tract infection by enteric pathogens. Another plausible mechanism is the anti-inflammatory activity of PPIs. Previous studies have suggested that PPIs inhibit neutrophil functions such as chemotaxis, 
superoxide production, and degranulation, ${ }^{33}$ which may increase host susceptibility to infection. To understand the exact mechanism, further evaluation of gut microbial change is needed.

The duration-response effect has been inconsistently demonstrated in several PPI studies. ${ }^{10,34,35}$ Due to the heterogeneity between studies, the effect was difficult to interpret. In this study, there was no clear duration-response relationship between PPI and PLA. The plausible reasons are follows: (1) the number of events is small, (2) the dose of PPI may be high even if the duration is short, and (3) the causal associations may be characterized by a threshold effect rather than a monotonic trend. ${ }^{2}$ In this case, the potential detrimental effect of PPI on PLA may not follow a simple duration-response effect and the trend of increasing exposure levels is not necessary.

Our data warrants careful interpretation as this is a retrospective study. The high PLA incidence in PPI users may not be from PPI itself, but from selection of symptomatic patients with liver abscess or biliary tract diseases. We excluded the first 7 days on PPI prescription from the definition of exposure periods to avoid bias. The relationship was remained significant. We also used multivariate and PS matching analyses to minimize heterogeneity between groups. In addition, it was difficult to analyze dose-response relationship between PPI and PLA because the ingredient and the dose of PPI for each drug were different. Furthermore, this study did not assess the causative organisms. Lastly, the study population comprised of Koreans and generalizability to other ethnicities and areas remains to be proven. Despite these limitations, the present study is less prone to bias than case-control studies. In addition, the strength of this data is large-cohort analysis with sufficient events and long-term follow-up.

In conclusion, the present data indicate that PPI use is associated with an increased PLA risk. Therefore, it is necessary to prescribe PPIs with clear indication and to avoid improper use of PPIs.

\section{Supplementary Materials}

Note: To access the supplementary tables mentioned in this article, visit the online version of Journal of Neurogastroenterology and Motility at http://www.jnmjournal.org/, and at https://doi. org/10.5056/jnm20221.

\section{Financial support: None.}

\section{Conflicts of interest: None.}

Author contributions: Joo Hyun Oh interpreted the data and prepared the draft of the manuscript; Danbee Kang collected, statistically analyzed, and interpreted the data and contributed to writing the manuscript; Wonseok Kang critically revised the manuscript; Eliseo Guallar designed the study, supervised the statistical analyses and interpretation of data, and critically revised the manuscript; Juhee Cho designed the study and statistically analyzed and interpreted the data; and Yang Won Min conceived and designed the study, and statistically analyzed and interpreted the data. Yang Won Min is guarantor of the article. All authors reviewed the manuscript for critical content and approved the final version.

\section{References}

1. Shin JM, Sachs G. Pharmacology of proton pump inhibitors. Curr Gastroenterol Rep 2008;10:528-534.

2. Vaezi MF, Yang YX, Howden CW. Complications of proton pump inhibitor therapy. Gastroenterology 2017;153:35-48.

3. Dial S, Delaney JA, Barkun AN, Suissa S. Use of gastric acid-suppressive agents and the risk of community-acquired Clostridium difficileassociated disease. JAMA 2005;294:2989-2995.

4. Kwok CS, Arthur AK, Anibueze CI, Singh S, Cavallazzi R, Loke YK. Risk of Clostridium difficile infection with acid suppressing drugs and antibiotics: meta-analysis. Am J Gastroenterol 2012;107:1011-1019.

5. Deshpande A, Pasupuleti V, Thota P, et al. Acid-suppressive therapy is associated with spontaneous bacterial peritonitis in cirrhotic patients: a meta-analysis. J Gastroenterol Hepatol 2013;28:235-242.

6. Kaplan GG, Gregson DB, Laupland KB. Population-based study of the epidemiology of and the risk factors for pyogenic liver abscess. Clin Gastroenterol Hepatol 2004;2:1032-1038.

7. Huang CJ, Pitt HA, Lipsett PA, et al. Pyogenic hepatic abscess. Changing trends over 42 years. Ann Surg 1996;223:600-607; discussion 607609.

8. Min YW, Kang D, Shin JY, et al. Use of proton pump inhibitors and the risk of cholangitis: a nationwide cohort study. Aliment Pharmacol Ther 2019;50:760-768.

9. Lin HF, Liao KF, Chang CM, Lin CL, Lai SW. Correlation between proton pump inhibitors and risk of pyogenic liver abscess. Eur J Clin Pharmacol 2017;73:1019-1025.

10. Wang YP, Liu CJ, Chen TJ, Lin YT, Fung CP. Proton pump inhibitor use significantly increases the risk of cryptogenic liver abscess: a population-based study. Aliment Pharmacol Ther 2015;41:1175-1181.

11. Lee J, Lee JS, Park SH, Shin SA, Kim K. Cohort profile: the national health insurance service-national sample cohort (NHIS-NSC), south Korea. Int J Epidemiol 2017;46:e15.

12. National Health Insurance Service (NHIS). National health examination statistical yearbook. Seoul: National Health Insurance Service, 2014.

13. Chun CB, Kim SY, Lee JY, Lee SY. Republic of Korea: health system review. Health Syst Transit 2009;11:1-184

14. Melzer M, Toner R, Lacey S, Bettany E, Rait G. Biliary tract infection and bacteraemia: presentation, structural abnormalities, causative organ- 
isms and clinical outcomes. Postgrad Med J 2007;83:773-776.

15. Lévesque LE, Hanley JA, Kezouh A, Suissa S. Problem of immortal time bias in cohort studies: example using statins for preventing progression of diabetes. BMJ 2010;340:b5087.

16. Shintani AK, Girard TD, Eden SK, Arbogast PG, Moons KG, Ely EW. Immortal time bias in critical care research: application of timevarying cox regression for observational cohort studies. Crit Care Med 2009;37:2939-2945.

17. Chen SC, Tsai SJ, Chen CH, et al. Predictors of mortality in patients with pyogenic liver abscess. Neth J Med 2008;66:196-203.

18. Shin DW, Cho B, Guallar E. Korean national health insurance database. JAMA Intern Med 2016;176:138.

19. Park TH, Choi JC. Validation of stroke and thrombolytic therapy in Korean national health insurance claim data. J Clin Neurol 2016;12:42-48.

20. Seo HJ, Oh IH, Yoon SJ. A comparison of the cancer incidence rates between the national cancer registry and insurance claims data in Korea. Asian Pac J Cancer Prev 2012;13:6163-6168.

21. Charlson ME, Pompei P, Ales KL, MacKenzie CR. A new method of classifying prognostic comorbidity in longitudinal studies: development and validation. J Chronic Dis 1987;40:373-383.

22. Sundararajan V, Henderson T, Perry C, Muggivan A, Quan H, Ghali WA. New ICD-10 version of the charlson comorbidity index predicted in-hospital mortality. J Clin Epidemiol 2004;57:1288-1294.

23. Yokota T, Iwamoto K, Watanabe Y, Yamauchi H, Kikuchi S, Hatori M. Pyogenic liver abscesses secondary to carcinoma of the sigmoid colon: a case report and clinical features of 20 cases in Japan. Ups J Med Sci 2005;110:241-244.

24. Abraham NS. Proton pump inhibitors: potential adverse effects. Curr Opin Gastroenterol 2012;28:615-620.

25. Shin JM, Kim N. Pharmacokinetics and pharmacodynamics of the proton pump inhibitors. J Neurogastroenterol Motil 2013;19:25-35.
26. Bavishi C, Dupont HL. Systematic review: the use of proton pump inhibitors and increased susceptibility to enteric infection. Aliment Pharmacol Ther 2011;34:1269-1281.

27. Lo WK, Chan WW. Proton pump inhibitor use and the risk of small intestinal bacterial overgrowth: a meta-analysis. Clin Gastroenterol Hepatol 2013;11:483-490.

28. Koo SH, Deng J, Ang DSW, et al. Effects of proton pump inhibitor on the human gut microbiome profile in multi-ethnic groups in Singapore. Singapore Med J 2019;60:512-521.

29. Bajaj JS, Cox IJ, Betrapally NS, et al. Systems biology analysis of omeprazole therapy in cirrhosis demonstrates significant shifts in gut microbiota composition and function. Am J Physiol Gastrointest Liver Physiol 2014;307:G951-G957.

30. Shi YC, Cai ST, Tian YP, et al. Effects of proton pump inhibitors on the gastrointestinal microbiota in gastroesophageal reflux disease. Genomics Proteomics Bioinformatics 2019;17:52-63.

31. Siu LK, Yeh KM, Lin JC, Fung CP, Chang FY. Klebsiella pneumoniae liver abscess: a new invasive syndrome. Lancet Infect Dis 2012;12:881887.

32. Yang YSH, Chang HW, Lin IH, et al. Long-term proton pump inhibitor administration caused physiological and microbiota changes in rats. Sci Rep 2020;10:866.

33. Wandall JH. Effects of omeprazole on neutrophil chemotaxis, super oxide production, degranulation, and translocation of cytochrome b-245. Gut 1992;33:617-621.

34. Vestergaard P, Rejnmark L, Mosekilde L. Proton pump inhibitors, histamine $\mathrm{H} 2$ receptor antagonists, and other antacid medications and the risk of fracture. Calcif Tissue Int 2006;79:76-83.

35. Kaye JA, Jick H. Proton pump inhibitor use and risk of hip fractures in patients without major risk factors. Pharmacotherapy 2008;28:951-959. 